\title{
Conjugated linoleic acids: all the same or to everyone its own function?
}

\author{
Jean-Charles MARTIN*, Karine VALEILlE \\ Laboratoire de Physiologie de la Nutrition, Université de Paris-Sud, 91400 Orsay, France
}

(Received 14 June 2002; accepted 6 November 2002)

\begin{abstract}
Conjugated linoleic acid (CLA) is a generic term referring to a mixture of geometrical and positional isomers of linoleic acid in which up to 16 members have been identified. Many potentially beneficial health effects have been ascribed to these fatty acids when consumed as a mixture, and where generally 2 isomers dominate, e.g. the $9 \mathrm{c}, 11 \mathrm{t}$-isomer, the so-called rumenic acid, and the 10t,12c-isomer: anti-carcinogenic, immune modulator, anti-atherosclerotic, and anti-obesity among the most spectacular. The question arises as to whether the pleiotropic biological activity is supported by one or several of the isomers. Recent studies using pure individual isomers have started to elucidate this issue, but many others are required to ascribe a respective role to each CLA isomer (the main ones as well as the minor ones), such as those occurring in some complex mixtures already commercially available, or even in foodstuff. The aim of the present study was to focus on the CLA-isomer specific effects depicted in the literature up to now.
\end{abstract}

CLA isomers / rumenic acid / cancer / obesity / atherosclerosis

\section{INTRODUCTION}

A great deal of concern has arisen from the study of conjugated linoleic acids (CLA), because of their considerable pleiotropic effects: anti-carcinogenic, immune modulator, anti-diabetic, anti-obesity, anti-thrombotic and anti-atherogenic $[4,5,47]$. CLA are 18 carbon chain-length fatty acids with 2 double bonds. These are therefore isomers of linoleic acid, but to the contrary to this essential fatty acid where the double bonds are methylene-interrupted, they are consecutive (e.g. conjugated) in CLA (Fig. 1). The double bond system is localized on carbons 7,$9 ; 8,10$, etc. up to carbon 12,14 of the

* Correspondence and reprints

E-mail: jean-charles.martin@ibaic.u-psud.fr 


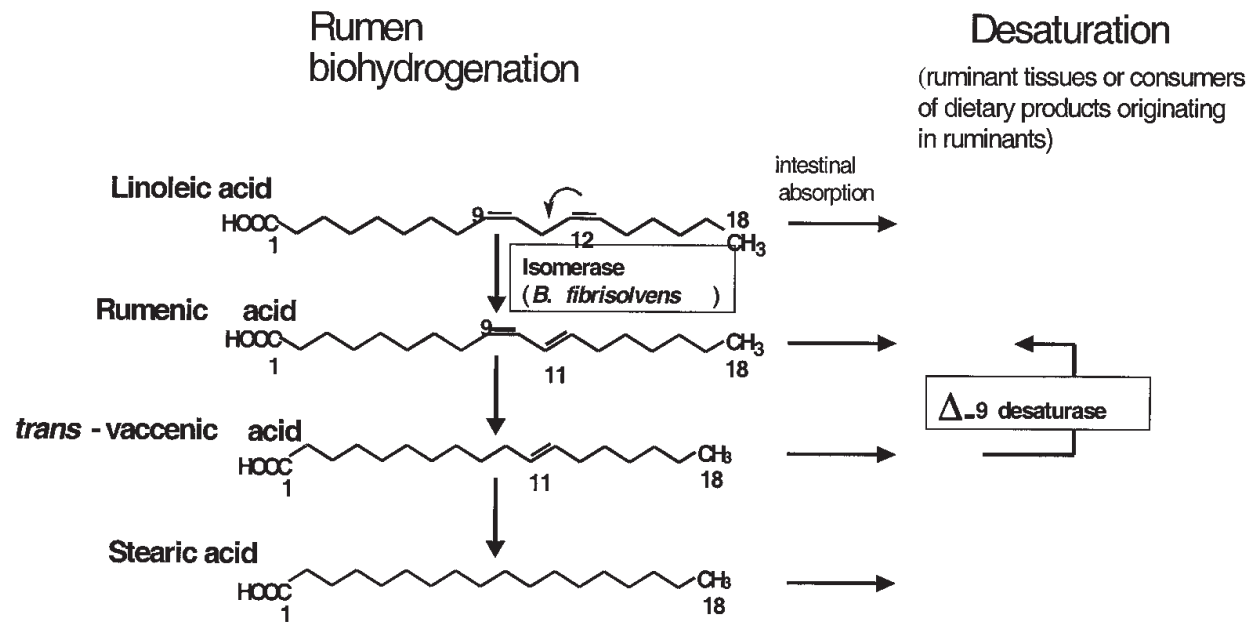

Figure 1. Natural origin of rumenic acid: rumenal synthesis from linoleic acid, and endogenous occurrence from delta-9 desaturation of trans-vaccenic acid (from [26], with permission).

olefinic chain, including all possible geometrical combinations (cis/cis, cis/trans, trans/cis et trans/trans). As many as 16 members have been identified thus far in marketed products [38]. The latter are obtained by alkaline isomerization of vegetable oil enriched with linoleic acid (safflower oil, sunflower oil) and sold as food supplements. In this example, the CLA isomeric distribution is generally dominated by 2 main isomers, e.g. the $9 c, 11 t$-isomer and $10 t, 12 c$-isomer, including in some preparations the $8 t, 10 c$ - and $11 t, 13 c$-isomers. Conversely, the $9 c, 11 t$-isomer is the main CLA occurring naturally in foodstuff (up to $80 \%$ of total isomers) [23,43], although the other isomers are also present in minor amounts and should therefore be considered as "natural" compounds [43]. Importantly, the balance between the various isomers is not the same in synthetic CLA products and those occurring from natural sources. Since most of the CLA intake and therefore the $9 c, 11 t$ isomer arise from ruminant products, this isomer is called rumenic acid. It is a byproduct of microbial biohydrogenation that takes place in the rumen from linoleic acid (and $\alpha$-linolenic acid) occurring from plants and ingested by ruminants (Fig. 1). Some of the rumenic acid formed escapes total hydrogenation and is taken up by the intestines and reaches milk and muscle lipids. Transvaccenic acid (18 carbons long, one transdouble bond located in the $\Delta-11$ position, another by-product of the biohydrogenation reaction), can also undergo delta-9 desaturation in the intestines, liver, mammary gland, and adipose tissue, and there it forms rumenic acid endogenously [25] (Fig.1). In humans, trans-vaccenic acid occurring from the intake of ruminant products can be similarly converted to the $9 c, 11 t$-isomer [ 1 , 48], This comes in addition to the daily 200-400 mg of pre-formed ingested rumenic acid [15].

When dealing with the bioactivity of CLA, it is likely that their structural peculiarities underly some of their radically different actions when compared to linoleic acid (reviewed in [4, 47]). Nevertheless, only a few studies have addressed the discrete potency of each isomer (Tab. I), or the particular synergistic or competitive isomers-effects of several isomers present together in the same mixture. 
Biological effect of CLA isomers

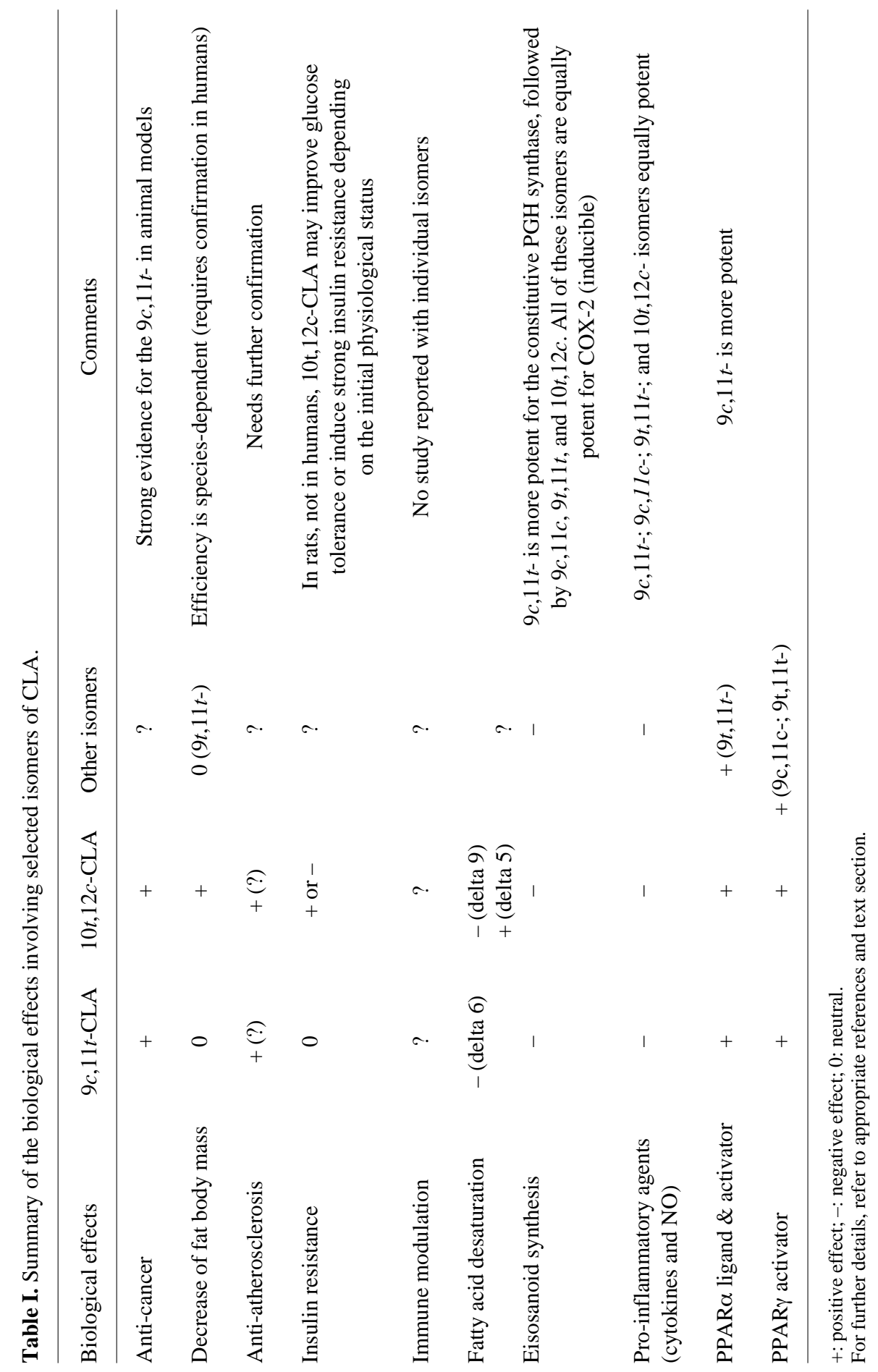




\section{CANCER AND CLA: WHICH ISOMER IS POTENT?}

Fifteen years ago Pariza et al. [17] found that CLA of a fried ground beef extract was highly potent in reducing epidermal tumor incidence of mice topically treated with 12-O-tetradecanoylphorbol-13-acetate. This suggests that the anticarcinogenic effect was due to one isomer, since the CLA in ruminant products are mainly made up of $9 c, 11 t$-CLA. The potency of this isomer has been further confirmed by us [22] and Ip [19] in another carcinogenic model, e.g. NMU-induced rat mammary carcinogenesis. In these experiments, female rats were injected with a pro-carcinogen and fed for 6 months with diets containing either a CLA mixture (complex mixture), or a chemicallyprepared $9 c, 11 t$-CLA [22], or a butter diet naturally enriched in the $9 c, 11 t$-CLA, or artificially increased with a CLA mixture (Ip study). In both experiments, CLA amounted to $1 \%$ by weight. The cancer risk was decreased in all CLA-diets, including those enriched with only the $9 c, 11 t$-isomer, thereby indicating a high anticarcinogenic potency for this isomer, the so-called rumenic acid. In addition, a recent casecontrol study carried out on Finish women [3] demonstrated that breast cancer risk is decreased by 2.5 fold (odds ratio of 0.4 ) in the women with the highest serum $9 c, 11 t$-CLA concentrations as compared to those with the lowest concentrations. On the contrary, in vitro studies have also demonstrated that the $10 t, 12 c$-isomer is even more potent than the $9 \mathrm{c}, 11 \mathrm{t}$-isomer against colorectal cancer proliferation [33]. In conclusion, there is a strong indication that the $9 c, 11 t$-isomer has an anticarcinogenic effect; there is also a good indication that the $10 t, 12 c$ isomer is also a potent anticarcinogen.

\section{CLA ISOMERS IN THE MANAGEMENT OF FAT BODY MASS}

There is now strong evidence showing that the $10 t, 12 c$-isomer is mostly responsi- ble for the fat reduction observed upon CLA treatment [34]. This was demonstrated effectively by a mouse study in which different purified isomers were used $(10 t, 12 c-, 9 c, 11 t$, $9 t, 11 t)$ and in which the $10 t, 12 c$-isomer was the most efficacious in decreasing body fat mass [35]. According to Pariza [34], CLA and specifically the $10 t, 12 c$-isomer blocks body fat gain, but does not necessarily reduce the body fat level which had accumulated prior to the CLA administration (Fig. 2).

We reached the same conclusion in our experiments. We fed hamsters a lipidenriched diet (33\% in energy) for 8 weeks, supplemented or not with CLA (1\% by weight). Only the CLA diet containing the $10 t, 12 c$-isomer prevented the accretion of body triglyceride over time, while the diet containing the sole $9 c, 11 t$-isomer failed to do so [8]. A recent human study examined the effects of feeding obese men with the metabolic syndrome for 12 weeks $3.4 \mathrm{~g}$ of either $10 t, 12 c$-CLA, or a CLA mixture containing equal amounts of both the $9 c, 11 t$ and 10t,12c-CLA [40]. The sagittal abdominal diameter and \% body fat (determined by bioelectrical impedance analysis) decreased similarly in both CLA groups compared to the baseline values, but not compared to the placebo values at the completion of the study. It is noteworthy that the treatment with $10 t, 12 c$-CLA, but not with the CLA mixture, worsened the insulin sensitivity in that population. Other human data performed on populations free of the metabolic syndrome have reported the use of more or less complex mixtures in which the bioactive isomer in fat management, e.g. the $10 t, 12 c$-isomer, is diluted among the other isomers $(10 t, 12 c$-isomer ranging from less than 20\% [52] to up to $45 \%$ [44] of the overall CLA isomers). This variable dilution of the $10 t, 12 c$-isomer among the preparations might explain the fact that some authors have found an effective but moderate fat reduction upon CLA supplementation [29, 39, 44], whereas others have failed [52]. Taken together, these studies indicate 




Figure 2. Putative mechanisms underlying fat reduction by CLA (from [34], with permission). LPL: lipoprotein lipase; $\Delta 9$ desat: delta- 9 desaturase.

that the $10 t, 12 c$-isomer is likely the biologically active isomer in fat management in humans, but its effect is more modest than that observed in animal models such as mice. Also, the other isomers present in the most complex mixtures are apparently not effective in reducing body fat.

In conclusion, there is strong evidence showing that the $10 t, 12 c$-isomer is the active isomer in eliciting body fat reduction, whereas $9 c, 11 t$ is not. A more precise evaluation of the $10 t, 12 c$-isomer on populations free of the metabolic syndrome would help to more precisely define the efficiency of CLA in body fat management.

\section{CLA ISOMERS AND ATHEROSCLEROSIS}

There are four important well-recognized causes leading to a pejorative atherosclerotic phenotype: (1) the plasma lipid profile, (2) the lipid deposition in vessels, (3) the platelet aggregation feature, and (4) the inflammatory status in the arterial wall.

\subsection{CLA isomers and lipid status}

A hamster study in which the $10 t, 12 c$ or the $9 c, 11 t$-isomer ( $0.66 \%$ by weight) were exchanged for linoleic acid in the diet revealed that only the $10 t, 12 c$-CLA (or a CLA mixture containing this isomer) decreased the fasting value of LDL- and HDL-cholesterol (18 and 11\%, [13]) and increased VLDL-TG (61\%), while the $9 c, 11 t$-isomer did not display such an effect. On the contrary, we demonstrated that when either the $9 c, 11 t$-isomer $(0.5 \%$ by weight $)$ or a CLA mixture $(10 t, 12 c$ - \& $9 c, 11 t$-isomer, $50: 50,1 \%$ by weight) were added to a lard based-diet without any substitution for linoleic acid, only the $9 c, 11 t$ - additioned diet favorably increased the HDL-cholesterol $(32 \%)$ as well as the HDL-/LDLcholesterol ratio $(55 \%)$, with no modification of the VLDL-TG content $(+268 \%$ on the contrary for the CLA mixture) (unpublished results). The apparent discrepancy between the aforementioned studies may arise from the strain of hamsters used $\left(\mathrm{F}_{1} \mathrm{~B}\right.$ in one case, LPN Golden Syrian in the other study), the based-diet, and the way CLA were added 
to it (substitution of linoleic acid with CLA in one case, simple addition in the other one with no substitution), or a combination of the above reasons. In addition, it should be said that in one case the cholesterol content of the based-diet amounted to $0.01 \%$ (by weight), whereas it reached up to $0.06 \%$ in the second study, which makes the comparison of the studies difficult. With this in mind, in our study both CLA diets $(9 c, 11 t$ and CLA mix) increased the lipoprotein scavenger receptor mass (e.g. SR-BI and LDL-r) in a similar manner in the liver. Thus, from our data it appeared that the $9 c, 11 t$-isomer is potent in reducing the atherosclerotic risk, whether provided alone or together with the $10 t, 12 c$-isomer in a mixture. Although the CLA mixture appears effective in preventing aortic lipid deposition and in increasing fatty streak regression in both hamster and rabbit models [21, 50] (but not in mice [32]), there are no in vivo studies so far dealing with this specific issue while using individual isomers in animals. An in vitro study examined the lipid secretion by a human hepatocyte-like cell line (HepG2) treated with either $9 c, 11 t$ - or $10 t, 12 c$-CLA [24]. The VLDL-TG secretion rate was decreased only by the $10 t, 12 c$ CLA treatment. Nevertheless, this is not consistent with the observation of Riserius et al. [40] in obese humans with the metabolic syndrome, in which on the contrary, the VLDL-TG increased while feeding $3.4 \mathrm{~g}$ of the $10 t, 12 c$-CLA for 12 weeks. Feeding a CLA mixture induced no changes at the same time. This is consistent with other human studies using a CLA mixture which constantly demonstrate no changes in VLDLTG in populations free of the metabolic syndrome $[6,7,44]$. Also importantly, in the population with the metabolic syndrome, HDL-C decreased in the $10 t, 12 c$-CLA group [40], which together with the rise in VLDL-TG do not appear favorable in the etiology of atherosclerosis. Therefore, the studies using purified isomers give inconsistent results, probably because both the models and the experimental settings were different. More definitive conclusions as to the effect of the various CLA isomers on plasma lipids is therefore certainly required to compare experiments designed with a common background, such as animal model / population, CLA amounts and isomer profiles, metabolic status, ...

\subsection{CLA isomers and platelet aggregation}

Both the $9 c, 11 t$ - and $10 t, 12 c$-isomers are highly potent in inhibiting the calcium ionophore, arachidonic acid- and collageninduced Human platelet aggregation compared to linoleic acid [45]. In addition, they both inhibit the formation of the pro-aggregatory cyclooxygenase-catalyzed product, $\mathrm{TxA}_{2}$ [45]. Therefore, both isomers seemingly possess similar antithrombotic properties, at least in vitro, on human platelets.

\subsection{CLA isomers, inflammation and macrophage differentiation}

In addition, these isomers may also individually inhibit the formation of the proinflammatory prostaglandin PGF $2 \alpha$ by $40 \%$ in human saphenous vein endothelial cells stimulated by a calcium ionophore [49]. This can also contribute favorably to an antiatherogenic effect.

In an in vitro study using murine RAW macrophages [51], the authors also found that in this cell line, the $9 c, 11 t-; 9 c, 11 c$-; $9 t, 11 t-$; and $10 t, 12 c$ - isomers all identically decreased the production of the pro-inflammatory nitric oxide (NO) through inhibition (in a dose dependent manner) of the inducible nitric oxide synthase (iNOS) mRNA and iNOS gene promotor activity. In addition, they also decreased the production of TNF $\alpha$ by these macrophages, both at the protein and mRNA level, as well as the production of IL- $1 \mathrm{~b}$, and IL- 6 . These latter anti-inflammatory effects might be seen as favorable in the etiology of atherosclerosis. 
On the contrary, the 9,11-isomers only $(9 c, 11 t-; 9 c, 11 c-$; and $9 t, 11 t-)$ were all potent in increasing the differentiation of HL60 cells into monocytes and macrophages [51], which seems contradictory with their antiatherogenic role, since macrophages may ultimately lead to foam cell formation. Nevertheless, further studies are required to determine the net effect of CLA isomers among the anti-inflammatory role and macrophage differentiation on the pathogenesis of atherosclerosis.

In conclusion, both the $9 c, 11 t$ - and the $10 t, 12 c$ - isomers of CLA can be efficient in modulating the severity of atherosclerosis, either at the circulating lipid level or at the thrombotic and endothelial levels. On the contrary, all of the CLA isomers tested indistinctively decreased the level of pro-inflammatory agents produced in cell culturedmacrophages, whereas the 9,11-isomers only, and not the $10 t, 12 c$-isomer, induced macrophage differentiation. No human studies evaluating these potencies in vivo are available so far for the individual isomers.

\section{CLA ISOMERS}

\section{AND INSULIN RESISTANCE}

In the mouse model, the ingestion of a CLA mixture has been reported to increase insulin secretion and to decrease glucose clearance, leading to the paroxystic conditions of the lipodystrophic syndrome [46]. This effect has been unambiguously ascribed to the $10 t, 12 c$-isomer [12] in female mice. Paradoxically, this is not the case in the diabetic fa/fa obese Zucker rat (ZDF) in which CLA administrated as a mixture improved glucose tolerance [41] as much as the antidiabetic drugs, thiazolinediones [18]. We observed that hamsters fed with a CLA mixture containing only the $9 c, 11 t$ - and the $10 t, 12 c$-isomer (50:50 mixture) displayed an insulin resistance phenotype, whereas those fed with the $9 c, 11 t$-isomer did not (Unpublished results). In ZDF rats, only the 50:50 isomeric mixture is able to improve glucose tolerance, whereas the $9 c, 11 t$-isomer alone fails [41]. Therefore, in the animal model it seems that the $10 t, 12 c$-isomer is responsible for both insulin resistance and glucose tolerance according to the basal metabolic feature of the animal, and that the $9 c, 11 t$-isomer is neutral. Human studies published thus far have not reported insulin resistance following supplementation with the CLA mixture. Nevertheless, this is not the case in a recent study comparing the effect of the $10 t, 12 c$-isomer to that of a CLA mixture on insulin-resistant obese humans, where the authors observed a $19 \%$ loss of insulin sensitivity merely in the $10 t, 12 c$ treated individuals compared to the placebo [40]. In conclusion, the $10 t, 12 c$-isomer seems to support the insulin-resistant phenotype even in humans. Nevertheless, such an effect is not observed when this isomer is included in a mixture [40]. One could underline the great care that should be taken both in the consumption of CLA by a selected population, and in the choice of the CLA isomer used, as well as in the interaction between both.

\section{BIOAVAILABILITY OF CLA ISOMERS}

\subsection{Intestinal availability}

One of the first questions when dealing with nutrients is their bioavailability. There is seemingly no selectivity in the intestinal absorption of either CLA isomers, at least when ingested as triacylglycerol [27]. Especially, all the geometrical isomers of 9,11and 10,12-CLA displayed an identical lymph recovery, similar to that of linoleic acid.

\subsection{Esterification in complex lipids and cell processing}

This is not the case for their incorporation into complex lipids. In most cases, when a 
mixture of CLA is fed (up to 13 different isomers), the $9 c, 11 t$-isomer is almost always the main isomer found in tissue lipids, and the $10 t, 12 c$-isomer the least one [4, 20, 47]. A noticeable exception is in the heart phospholipids where the $11 c, 13 t$-isomer greatly accumulates over the other isomers and over its relative content in the diet [20]. Also, when rats were given either $9 c, 11 t-, 9 t, 11 t$, $10 t, 12 c-$, or $10 t, 12 t$ - as TG and for 6 days, the 9,11 isomers generally accumulated in higher amounts in tissue lipids (liver, kidneys, brain, retroperitoneal adipose tissue and heart) than their 10,12 homologues [2]. These observations partly rely on differences in the post-absorptive metabolism among the isomers. This seems to be the case for the oxidative degradation pathways in cell peroxisomes. For instance, the $9 c, 11 t$-isomer oxidative breakdown is lower in these organites than for the $10 t, 12 c$ isomer [16, 28]. This lesser breakdown would explain why the $9 c, 11 t$-isomer is found esterified in higher amounts in complex lipids (triacylglycerol mainly). In addition, if indeed most of the CLA might be oxidized or esterified, a small amount may undergo another metabolic fate: whereas the $9 c, 11 t$-isomer can be desaturated and chain-elongated, $10 t, 12 c$-CLA can be chain-shortened in the peroxisomes into a 16 carbon chain length conjugated fatty acid, or else simply delta- 6 desaturated merely to get a conjugated isoform of linolenic acid [42] (Fig. 3). It is of course likely that all of these metabolites can be biologically potent $[4,34]$.

\section{MODULATION OF CELL METABOLISM BY SELECTED ISOMERS OF CLA}

\subsection{Fatty acid desaturation}

Interestingly, both the $9 c, 11 t$ - and the $10 t, 12 c$-CLA can interfere with the metabolism of the other fatty acids through modulation of the desaturase activities. However, the in vitro delta- 6 desaturase activity is preferentially decreased by the $9 c, 11 t$-isomer $[9,11]$, with no change in the delta-6 mRNA expression [11]. On the contrary, the ingestion of the $10 t, 12 c$-isomer by rats (male Sprague Dawley) seems to increase the apparent delta-5 desaturation activity in the liver microsomes [42]. As a result, the $\mathrm{C} 22$ polyunsaturated fatty acid content in the liver membrane increases [42]. Moreover, the $10 t, 12 c$-isomer is able to decrease the in vitro and in vivo (apparent) delta- 9 desaturase activity $[9,42]$ and gene expression [36], leading to less oleic acid



Figure 3. Cellular metabolism and bioavailability of the 2 main CLA isomers. 
in tissue lipids. On the contrary, the human hepatocyte-like cell line (HepG2) treated with the $10 t, 12 c$-isomer have reduced apparent $\Delta 9, \Delta 6$, and $\Delta 5$ desaturase activities, whereas those treated with the $9 c, 11 t$-isomer have only reduced apparent $\Delta 9$ activity [14]. Briefly, both isomers have a distinct effect on the desaturation of MUFA and PUFA, with the 10t,12c-isomer consistently reducing the $\Delta 9$ desaturase. This, might be consequential to modulate the synthesis of eicosanoids as well as membrane fluidity and peroxydability. A decrease of the estimated $\Delta 6$ and $\Delta 9$ activities and an increase in the $\Delta 5$ desaturase one were observed in humans consuming a CLA mixture [44]. Most generally, the biological consequence of the modification of the fatty acid profile by CLA isomers should be more thoroughly investigated.

\subsection{Synthesis of eicosanoids}

In addition to modulating the availability of the parent fatty acids involved in eicosanoid synthesis, CLA might also inhibit the in vitro prostaglandin synthesis through inhibition of the constitutive cyclooxygenase prostaglandin $\mathrm{H}$ synthase (PGHS) of Ram seminal vesicles. The $9 c, 11 t$-isomer appears to be the most effective, followed by the $9 c, 11 c-$, the $10 t, 12 c-$, and finally the $9 t, 11 t$-isomers [10]). A related finding has been observed with the inducible cyclooxygenase (COX-2 of the PGHS-2 complex) of cultured murine RAW macrophages [51], where several CLA isomers (e.g. 9c,11t-; $9 c, 11 c-; 9 t, 11 t-;$ and $10 t, 12 c-)$ all identically decreased the mRNA expression of this enzyme. Nonetheless, it should be said that linoleic acid displayed the same effect. Consistent with the mRNA expression, the reaction product of COX-2, $\mathrm{PGE}_{2}$, decreased in a dose dependent manner along with $9 c, 11 t$ - administration [51]. These observations are of major concern, since eicosanoids and especially prostaglandins are involved in most of the effects in which CLA are also potent (e.g. carcinogenesis, atherosclerosis, obesity, immune function). This could contribute to explain some differences in the biological activity of individual CLA isomers.

\subsection{Modulation of gene expression by selected CLA isomers through transcription factors}

It has also been reported that individual CLA isomers are able to modulate gene expression since they can bind and activate transcription factors such as PPARs [30, 31, 51]. CLA isomers were shown to be ligands for human PPAR $\alpha$ with a rank order of potency of $9 c, 11 t->10 t, 12 c->9 t, 11 t$-, with the $9 c, 11 t$-isomer being the most efficacious PPAR $\alpha$ activator in a transfected cell model (COS-1) [31]. It was demonstrated that the $9 c, 11 t$-isomer was as potent as $\mathrm{Wy}-14,643$, a well-known peroxisome ligand and activator, to activate PPAR $\alpha$ [30], consistent with the results of others [12]. On the contrary, CLA isomers do not always demonstrate a phenotypic feature of current peroxisome proliferators, such as in rats $[28,30]$ or hamsters [13] for instance. In addition, although CLA isomers and especially the $9 c, 11 t$-isomer are potent ligands and activators of PPARs, their lipid lowering effect still remains in PPAR $\alpha$ knock-out mice [37]. This indicates that the biological activities of either CLA isomers cannot be only ascribed to their activation of PPARs, and especially PPAR $\alpha$. The PPAR $\gamma$ pathway is another pathway which is affected by CLA, as found in cultured murine RAW macrophages [51]. In this example, several isomers of CLA $(9 c, 11 t-; 9 c, 11 c-; 9 t, 11 t-; 10 t, 12 c$-CLA) shown the same PPAR $\gamma$ activation properties in these cells, which account for the above reported effects in the production of the proinflammatory agents by macrophages, as well as macrophage differentiation. Other pathways regulated by many other transcription factors need to be explored (such as C/EBP, LXR, HNF4 $\alpha$, SREBP, NFкB) using the individual isomers of CLA to get 
a clearer picture of the effect of CLA on these important aspects of gene regulation.

\section{CONCLUSION}

A summary of the main effects of the individual isomers thus far evaluated is listed in Table I. Of course, these effects in the selected issues reported need to be further explored, especially the effects of the individual isomers on gene expression. In addition, most of the published results deal with both the $9 c, 11 t$ - and $10 t, 12 c$-isomers, and explain many of the already described effects obtained with complex mixtures of CLA. From this data, the claim that the $9 c, 11 t$-isomer is the only biologically active CLA is not supported. In addition, some distinct activities related to selected isomers are now appearing. Nonetheless, other isomers need to be evaluated, such as $11 c, 13 t$ sometimes present in high amounts in commercial preparations, or the $7 c, 9 t$-, present in dairy products, the $10 t, 12 t$ - formed in heated oil, etc. The limitation is the cost of the marketed products. Also, the efficiency of individual isomers in humans must be assessed so that the respective role of CLA in health as well as their possible toxicological effects are known. Such an approach could help to delineate the balance between the risk and the desired benefit while consuming either isomer, with the knowledge of the possible side-effects.

\section{ACKNOWLEDGEMENTS}

Dr Amir Ravandi is fully acknowledged for his language expertise.

\section{REFERENCES}

[1] Adlof R.O., Duval S., Emken E.A., Biosynthe sis of conjugated linoleic acid in humans, Lipids 35 (2000) 131-135.

[2] Alasnier C., Berdeaux O., Chardigny J.M., Sebedio J.L., Fatty acid composition and conjugated linoleic acid content of different tissues in rats fed individual conjugated linoleic acid isomers given as triacylglycerols, J. Nutr. Biochem. 13 (2002) 337-345.

[3] Aro A., Mannisto S., Salminen I., Ovaskainen M.L., Kataja V., Uusitupa M., Inverse association between dietary and serum conjugated linoleic acid and risk of breast cancer in postmenopausal women, Nutr. Cancer 38 (2000) 151-157.

[4] Banni S., Martin J.C., Conjugated linoleic acid and metabolites, in: Christie W.W., Sébédio J.L. (Eds.), Trans fatty acids in human nutrition, The Oily Press, Dundee, Scotland, 1998, pp. 261-302.

[5] Belury M.A., Dietary conjugated linoleic acid in health: Physiological effects and mechanisms of action, Annu. Rev. Nutr. 22 (2002) 505-531.

[6] Benito P., Nelson G.J., Kelley D.S., Bartolini G., Schmidt P.C., Simon V., The effect of conjugated linoleic acid on plasma lipoproteins and tissue fatty acid composition in humans, Lipids 36 (2001) 229-236

[7] Blankson H., Stakkestad J.A., Fagertun H., Thom E., Wadstein J., Gudmundsen O., Conjugated Linoleic Acid Reduces Body Fat Mass in Overweight and Obese Humans, J. Nutr. 130 (2000) 2943-2948

[8] Bouthegourd J.C., Even P. C., Gripois D., Tiffon B., Blouquit M.F., Roseau S., Lutton C., Tome D., Martin J.C., A CLA Mixture Prevents Body Triglyceride Accumulation without Affecting Energy Expenditure in Syrian Hamsters, J. Nutr. 132 (2002) 2682-2689.

[9] Bretillon L., Chardigny J.M., Grégoire S., Berdeaux O., Sébédio J.L., Effects of conjugated linoleic acid isomers on the hepatic microsomal desaturation activities in vitro, Lipids 34 (1999) 965-969.

[10] Bulgarella J.A., Patton D., Bull A.W., Modulation of prostaglandin $\mathrm{H}$ synthase activity by conjugated linoleic acid (CLA) and specific CLA isomers, Lipids 36 (2001) 407-412.

[11] Chuang L.T., Thurmond J.M., Liu J.W., Kirchner S.J., Mukerji P., Bray T.M., Huang Y.S., Effect of conjugated linoleic acid on fungal delta6desaturase activity in a transformed yeast system, Lipids 36 (2001) 139-143.

[12] Clement L., Poirier H., Niot I., Bocher V., Guerre-Millo M., Krief S., Staels B., Besnard P., Dietary trans-10,cis-12 conjugated linoleic acid induces hyperinsulinemia and fatty liver in the mouse, J. Lipid Res. 43 (2002) 1400-1409.

[13] de Deckere E.A., van Amelsvoort J.M., McNeill G.P., Jones P., Effects of conjugated linoleic acid (CLA) isomers on lipid levels and peroxisome proliferation in the hamster, Br. J. Nutr. 82 (1999) 309-317.

[14] Eder K., Slomma N., Becker K., Trans-10,cis-12 conjugated linoleic acid suppresses the desaturation of linoleic and alpha-linolenic acids in HepG2 cells, J. Nutr. 132 (2002) 1115-1121. 
[15] Fritsche J., Steinhart $\mathrm{H}$, Amounts of conjugated linoleic acid (CLA) in German foods and evaluation of daily intake, Food Res. Technol. 206 (1998) 77-82.

[16] Gurvitz A., Hamilton B., Ruis H., Hartig A., Hiltunen J.K., Degradation of conjugated linoleic acid isomers in the yeast Saccharomyces cerevisiae, Biochim. Biophys. Acta 1533 (2001) 81-85.

[17] Ha Y.L., Grimm N.K., Pariza M.W., Anticarcinogens from fried ground beef: heat-altered derivatives of linoleic acid, Carcinogenesis 8 (1987) 1881-1887.

[18] Houseknecht K.L., Vandenheuvel J.P., MoyaCamarena S.Y., Portocarrero C.P., Peck L.W., Nickel K.P., Belury M.A., Dietary conjugated linoleic acid normalizes impaired glucose tolerance in the Zucker diabetic fatty fa/fa rat, Biochem. Biophys. Res. Commun. 244 (1998) 678-682.

[19] Ip C., Banni S., Angioni E., Carta G., McGinley J., Thompson H.J., Barbano D., Bauman D., Conjugated linoleic acid-enriched butter fat alters mammary gland morphogenesis and reduces cancer risk in rats, J. Nutr. 129 (1999) 2135-2142.

[20] Kramer J.K.G., Sehat N., Dugan M.E.R., Mossoba M.M., Yurawecz M.P., Roach J.A.G., Eulitz K., Aaljus J.L., Schaefer A.L., Ku Y., Distributions of conjugated linoleic acid (CLA) isomers in tissue lipid classes of pigs fed a commercial CLA mixture determined by gas chromatography and silver ion-high performance chromatography, Lipids 33 (1998) 549-558.

[21] Kritchevsky D., Tepper S.A., Wright S., Tso P., Czarnecki S.K, Influence of conjugated linoleic acid (CLA) on establishment and progression of atherosclerosis in rabbits, J. Am. Coll. Nutr. 19 (2000) 472S-477S.

[22] Lavillonnière F., Rôle des acides gras diènes conjugués CLA dans la cancérogénèse mammaire. Recherche dans les produits laitiers, étude expérimentale chez le rat et dans le cancer du sein. Thèse de $3^{\mathrm{e}}$ cycle, Sciences de la vie, Université François Rabelais, Tours, 1998.

[23] Lavillonnière F., Martin J.C., Bougnoux P., Sébédio J.L., Analysis of conjugated linoleic acid isomers and content in French cheeses, J. Am. Oil Chem. Soc. 75 (1998) 343-352.

[24] Lin Y., Schuurbiers E., Van der Veen S., De Deckere E.A., Conjugated linoleic acid isomers have differential effects on triglyceride secretion in Hep G2 cells, Biochim. Biophys. Acta 1533 (2001) 38-46.

[25] Loor J.J., Lin X., Herbein J.H., Dietary transvaccenic acid (trans11-18:1) increases concentration of cis9,transll-conjugated linoleic acid (rumenic acid) in tissues of lactating mice and suckling pups, Reprod. Nutr. Dev. 42 (2002) 85-99.
[26] Martin J.C., Effet modulateur d'acides gras minoritaires atypiques dont les acides linoléiques conjugués sur le métabolisme lipidique, Cah. Nutr. Diét. 37 (2002) 86-94.

[27] Martin J.C., Sebedio J.L., Caselli C., Pimont C., Martine L., Bernard A., Lymphatic delivery and in vitro pancreatic lipase hydrolysis of glycerol esters of conjugated linoleic acids in rats, J. Nutr. 130 (2000) 1108-1114.

[28] Martin J.C., Gregoire S., Siess M.H., Genty M., Chardigny J.M., Berdeaux O., Juaneda P., Sebedio J.L., Effects of conjugated linoleic acid isomers on lipid-metabolizing enzymes in male rats, Lipids 35 (2000) 91-98.

[29] Mougios V., Matsakas A., Petridou A., Ring S., Sagredos A., Melissopoulou A., Tsigilis N., Nikolaidis M., Effect of supplementation with conjugated linoleic acid on human serum lipids and body fat, J. Nutr. Biochem. 12 (2001) 585-594.

[30] Moya-Camarena S.Y., Vanden Heuvel J.P., Belury M.A., Conjugated linoleic acid activates peroxisome proliferator-activated receptor a and b subtypes but does not induce hepatic peroxisome proliferation in Sprague-Dawley rats, Biochim. Biophys. Acta 1436 (1999) 331-342.

[31] Moya-Camarena S.Y., Vanden Heuvel J.P. Blanchard S.G., Leesnitzer L.A., Belury M.A., Conjugated linoleic acid is a potent naturally occuring ligand and activator of PPARa, J. Lipid Res. 40 (1999) 1426-1433.

[32] Munday J.S., Thompson K.G., James K.A. Dietary conjugated linoleic acids promote fatty streak formation in the C57BL/6 mouse atherosclerosis model, Br. J. Nutr. 81 (1999) 251-255.

[33] Palombo J.D., Gangulya A., Bistriana B.R., Menard M.P., The antiproliferative effects of biologically active isomers of conjugated linoleic acid on human colorectal and prostatic cancer cells, Cancer Lett. 177 (2002) 163-172.

[34] Pariza M.W., Park Y., Cook M.E., The biologically active isomers of conjugated linoleic acid, Prog. Lipid Res. 40 (2001) 283-298.

[35] Park Y., Storkson J.M., Albright K.J., Liu W . Pariza M.W., Evidence that the trans-10,cis12 isomer of conjugated linoleic acid induces body changes in mice, Lipids 34 (1999) 235-241.

[36] Park Y., Storkson J.M., Ntambi J.M., Cook M.E., Sih C.J., Pariza M.W., Inhibition of hepatic stearoyl-CoA desaturase activity by trans10,cis-12 conjugated linoleic acid and its derivatives, Biochim. Biophys. Acta 1486 (2000) 285-292.

[37] Peters J.M., Park Y., Gonzalez F.J., Pariza M.W., Influence of conjugated linoleic acid on body composition and target gene expression in peroxisome proliferator-activated receptor [alpha]-null mice, Biochim. Biophys. Acta 1533 (2001) 233-242. 
[38] Rickert R., Steinhart H., Fristche J., Sehat N Yurawecz M.P., Mossoba M.M., Roach J.A.G. Eulitz K., Ku Y., Kramer J.K.G., Enhanced resolution of conjugated linoleic acid isomers by tandem-column silver-ion high performance liquid chromatography, J. High Resolut. Chromatogr. 22 (1999) 144-148.

[39] Riserus U., Berglund L., Vessby B., Conjugated linoleic acid (CLA) reduced abdominal adipose tissue in obese middle-aged men with signs of the metabolic syndrome: a randomised controlled trial, Int. J. Obes. Relat. Metab. Disord. 25 (2001) 1129-1135.

[40] Riserus U., Arner P., Brismar K., Vessby B., Treatment with dietary trans-10,cis-12 conjugated linoleic acid causes isomer-specific insulin resistance in obese men with the metabolic syndrome, Diabetes Care 25 (2002) 1516-1521.

[41] Ryder J.W., Portocarrero C.P., Song X.M., Cui L., Yu M., Combatsiaris T., Galuska D., Bauman D.E., Barbano D.M., Charron M.J., Zierath J.R., Houseknecht K.L., Isomer-specific antidiabetic properties of conjugated linoleic acid. Improved glucose tolerance, skeletal muscle insulin action, and UCP-2 gene expression, Diabetes 50 (2001) 1149-1157.

[42] Sebedio J.L., Angioni E., Chardigny J.M., Gregoire S., Juaneda P., Berdeaux O., The effect of conjugated linoleic acid isomers on fatty acid profiles of liver and adipose tissues and their conversion to isomers of 16:2 and 18:3 conjugated fatty acids in rats, Lipids 36 (2001) 575-582.

[43] Sehat N., Kramer J.K.G., Mossoba M.M., Yurawecz M.P., Roach J.A.G., Eulitz K. Morehouse K., Ku Y., Identification of conjugated linoleic acid isomers in cheese by gas chromatography, silver ion high performance liquid chromatography and mass spectral reconstructed ion profiles. Comparison of chromatographic elution sequences, Lipids 33 (1998) 963-971.
[44] Smedman A., Vessby B., Conjugated linoleic acid supplementation in humans - metabolic effects, Lipids 36 (2001) 773-781.

[45] Truitt A., McNeill G., Vanderhoek J.Y., Antiplatelet effects of conjugated linoleic acid isomers, Biochim. Biophys. Acta 1438 (1999) 239-246.

[46] Tsuboyama-Kasaoka N., Takahashi M., Tanemura K., Kim H.J., Tange T., Okuyama H., Kasai M., Ikemoto S., Ezaki O., Conjugated linoleic acid supplementation reduces adipose tissue by apoptosis and develops lipodystrophy in mice, Diabetes 49 (2000) 1534-1542.

[47] Turini M., Martin J.C., Conjugated linoleic acid, in: Gunstone F.D. (Ed.) Structured lipids Marcel Decker, New York, 2001, pp. 251-284.

[48] Turpeinen A.M., Mutanen M., Aro A., Salminen I., Basu S., Palmquist D.L., Griinari J.M., Bioconversion of vaccenic acid to conjugated linoleic acid in humans, Am. J. Clin. Nutr. 76 (2002) 504-510.

[49] Urquhart P., Parkin S.M., Rogers J.S., Bosley J.A., Nicolaou A., The effect of conjugated linoleic acid on arachidonic acid metabolism and eicosanoid production in human saphenous vein endothelial cells, Biochim. Biophys. Acta 1580 (2002) 150-160.

[50] Wilson T.A., Nicolosi R.J., Chrysam M., Kritchevsky D., Conjugated linoleic acid reduces early aortic atherosclerosis greater than linoleic acid in hypercholesterolemic hamsters, Nutr. Res. 20 (2000) 1795-1805.

[51] Yu Y., Correll P.H., Vanden Heuvel J.P., Conjugated linoleic acid decreases production of pro-inflammatory products in macrophages: evidence for a PPAR[gamma]-dependent mechanism, Biochim. Biophys. Acta 1581 (2002) 89-99.

[52] Zambell K.L., Keim N.L., Van Loan M.D., Gale B., Benito P., Kelley D.S., Nelson G.J., Conjugated linoleic acid supplementation in humans: Effects on body composition and energy expenditure, Lipids 35 (2000) 777-782.

\section{List of abbreviations}

C/EBP: CAAT enhancer binding protein, CLA: conjugated linoleic acids, COX: cyclooxygenase, HDL: high density lipoproteins, HNF4 $\alpha$ : human nuclear factor 4 alpha, IL: interleukines, LDL: low density lipoproteins, LDL-r: VLDL/LDL receptor, LXR: liver X receptor, MUFA: monounsaturated fatty acids, NFкB: nuclear factor $\kappa B$, NMU: N-methyl-nitrosourea, NO: nitric oxide, PGE2: prostaglandin E2, PGF2 $\alpha$ : prostaglandin F2 $\alpha$, PGHS: prostaglandin H synthase, PPAR: peroxisome proliferator activated receptors, PUFA: polyunsaturated fatty acids, SR-BI: scavenger receptor type I, SREBP: sterol regulatory element binding protein, TG: triacylglycerols, TNF $\alpha$ : tumor necrosis factor alpha, TxA2: thromboxane A2, VLDL: very low density lipoproteins. 